\title{
Improvement of vibration control of a pneumatic shaker
}

\author{
Shouqiang Men \\ School of Science \\ Xian Technological University \\ Xian, China \\ e-mail: shqmen@yahoo.com \\ Jun Zhou \\ School of Science \\ Xian Technological University \\ Xian, China \\ e-mail:woxiaoxuechi@163.com
}

\author{
Jingwen $\mathrm{Xu}$ \\ Library \\ Xian Technological University \\ Xian, China \\ e-mail: jwenxu@sina.com
}

\begin{abstract}
A pneumatic shaker was exploited to excite Faraday instabilities in the study of interface movements of electrically conducting fluids. The frequency of the vibration was controlled by a computer precisely, whereas its amplitude was adjusted by a shock absorber roughly, which lead to some fluctuations in the amplitude of the shaker. In order to reduce the fluctuations, a rubber belt was used to tighten the lid and the body of the cylinder. During the study, a contacting displacement meter was applied to measure the amplitude of the vibration displacement, and a fibre-optic laser vibrometer was also used to measure the vibration velocity which could be integrated to obtain the displacement. It was found that the displacement fluctuation was decreased effectively, and the Faraday instabilities were generated more steadily.
\end{abstract}

Keywords- Vibration; pneumatic shaker; amplitude fluctuation; shock absorber; rubber belt

\section{INTRODUCTION}

Interfacial instabilities always occur in electromagnetic processing of materials, this phenomenon is so important in industrial applications that it has attracted much attention in recent years [1-8]. But, most of the researches are concentrated on theoretical study of the physical mechanisms since it is difficult to carry out experimental investigations due to severe environments for contacting probes [1-6].

In our paper, a model experiment was designed to simulate the interfacial instabilities in liquid metal. In the experiment, a pneumatic shaker was used to excite Faraday instabilities, the frequency of the shaker was controlled accurately, whereas the displacement of the cylinder lid was difficult to be controlled. A rubber damper was used firstly. However, the amplitude of the lid can only be controlled roughly because of the hard stroke. Hence, a rubber belt was added to tighten the lid and the body of the cylinder. In this case, the fluctuation of the amplitude was attenuated effectively, and the better condition to generate Faraday instabilities was realized.

a contacting distance meter and a single-point fiberoptic laser vibrometer, respectively. Whereas the velocity and displacement of the oscillating interface was difficult to be measured, a qualitative result was carried out using the single-point fibre-optic laser vibrometer due to a strong scatter of light by the convex interface.

\section{EXPERIMENTAL SETUP}

The experimental setup consists mainly of a cylinder to contain fluids, a base to fix the cylinder, a Festo pneumatic shaker [9-10], a contacting displacement meter, and a Polytec Laser-Doppler vibrometer. The cylinder is made of plexiglass which is transparent in order to illuminate the cylinder by light sheet and observe the interfacial instabilities optically. Besides, the cylinder is fixed on the lid, i.e., the top end of the pneumatic shaker. The inner diameter of the cylinder is $50 \mathrm{~mm}$ and its height is $100 \mathrm{~mm}$ Galinstan, a liquid metal alloy at room temperature, and potassium aqueous solution are used to study the interfacial instability, both of their depth are $50 \mathrm{~mm}$. Below the cylinder there is the pneumatic shaker whose vertical vibration is controlled by a computer so as to excite Faraday instabilities at the interface between the two fluids.

A contacting distance meter with a resolution of $1 \mu \mathrm{m}$ was applied to measure the displacement of the cylinder lid, and a Polytec single-point fibre-optic vibrometer based on Laser-Doppler effect was exploited to investigate the vibrating speed and displacement, respectively. The speed scale of the vibrometer can be set to be $1 \mathrm{~mm} / \mathrm{s}$ to $500 \mathrm{~mm} / \mathrm{s}$. 


\section{RESULTS AND DISCUSSIONS}

Firstly, a $10.0 \mathrm{~Hz}$ square-wave signal was generated by the computer, which was used to switch on/off the pneumatic shaker to vibrate vertically. Then displacements of the base were measured by the contacting displacement meter as shown in Fig. 1, which shows a square-like wave pattern with the peak-to-peak value of about $0.2 \mathrm{~mm}$. At the top and bottom of the wave, it is apparent that there are some sharp fluctuations which was induced by the absorber fixed at the bottom of the shaker.

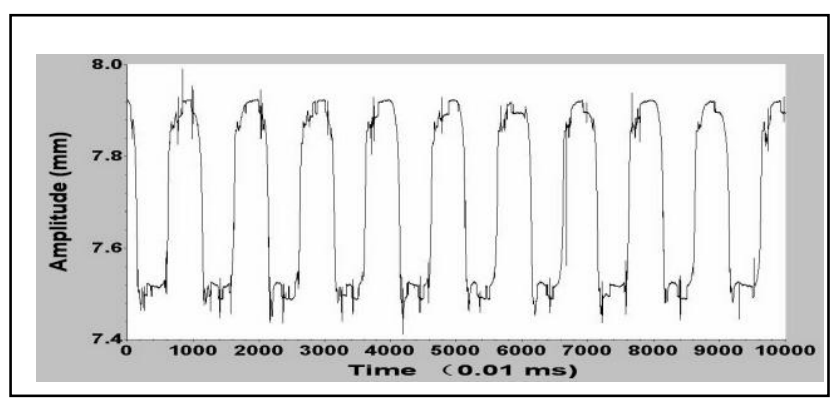

Figure 1. Displacement of the base with a shock absorber at a vibration frequency of $10.0 \mathrm{~Hz}$ measured by contacting displacement meter.

The amplitude of the cylinder lid is actually the stroke of the shaker which was controlled by the distance between the bottom end of the shaker and the shock absorber. It was not the originally fixed value of the shaker but could be adjusted conveniently.

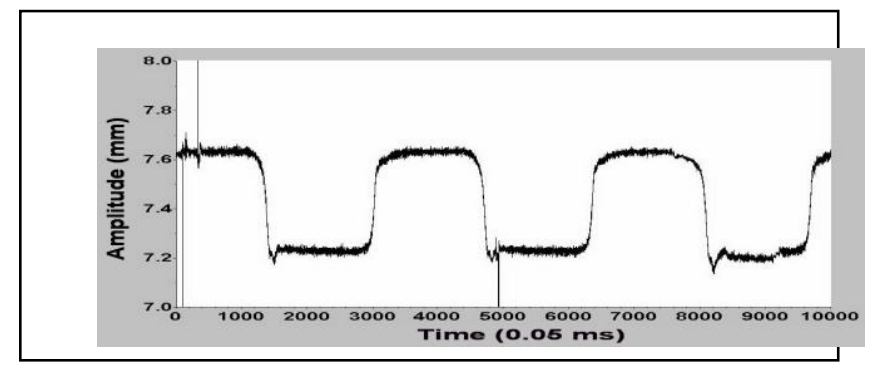

Figure 2 Displacement of the base with a shock absorber and a rubber belt used simultaneously at a vibration frequency of $6.0 \mathrm{~Hz}$ measured by contacting displacement meter.

In order to reduce the displacement fluctuations as much as possible, a rubber belt was applied to fasten the base or the piston rod and the bottom end of the pneumatic cylinder. Fig. 2 gives the detailed amplitude of the vibration amplitude of the cylindrical shaker when the shock absorber and a rubber belt were exploited simultaneously when a vibration frequency was fixed at $6.0 \mathrm{~Hz}$. It is clear that the fluctuations of the amplitude were decreased dramatically.

By optical observation, it was found that a surface wave pattern mode $(1,1)$ could be generated when the vibration frequency was in between $7.2-7.6 \mathrm{~Hz}$, where mode $(1,1)$ means there is only one peak along the azimuthal angle and there is also one peak in the radial direction as shown in Fig. 3. Hence, a mediate frequency $7.5 \mathrm{~Hz}$ and $7.3 \mathrm{~Hz}$ were chosen to be applied to excite Faraday instabilities for mode $(1,1)$ in the following studies, respectively.

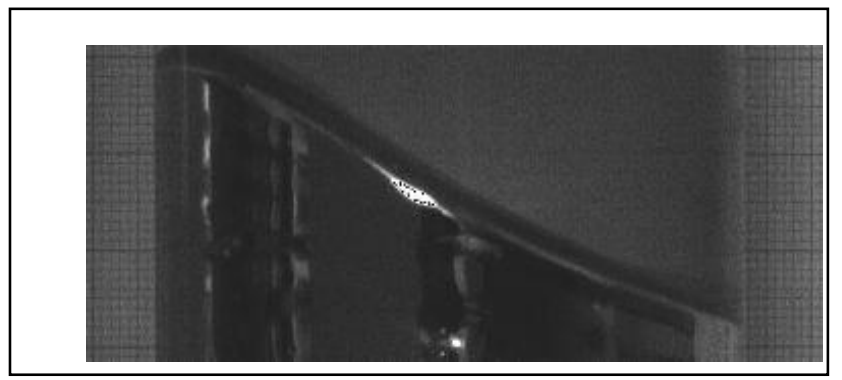

Figure 3 Image of surface wave mode $(1,1)$ at a vibration frequency of $7.5 \mathrm{~Hz}$

Figure 4 Displacement of the base with a shock absorber in

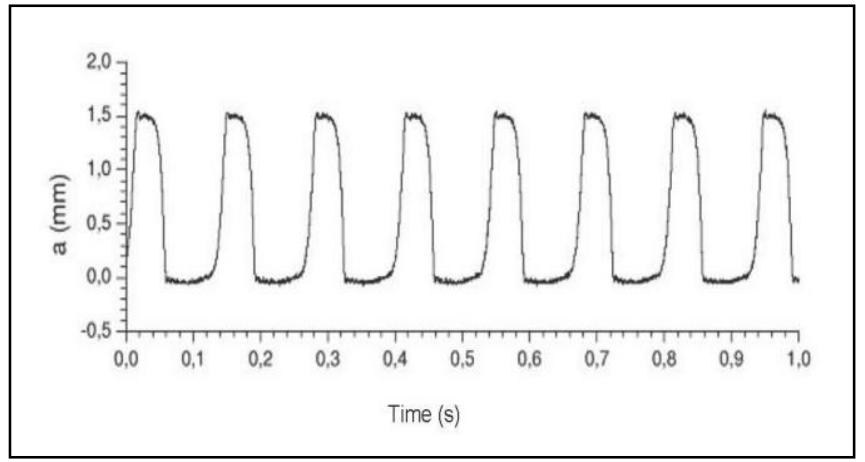

combination with a rubber belt at a vibration frequency of $7.5 \mathrm{~Hz}$ measured by contacting displacement meter.

The displacement of the base, in other words, the displacement of the cylinder lid, sometimes also called the displacement of the piston rod, was depicted versus time in one second as can be seen in Fig. 4, and the exciting frequency was fixed at $7.5 \mathrm{~Hz}$ as mentioned before. Although there are some ripples in the amplitude of the displacement, there aren't sudden, sharp noises, it became rather perfect in the periodicity, and the excited surface wave pattern, i.e., the mode $(1,1)$ became more stable.

Figure 5 FFT of the displacement excited by a $7.5 \mathrm{~Hz}$ signal

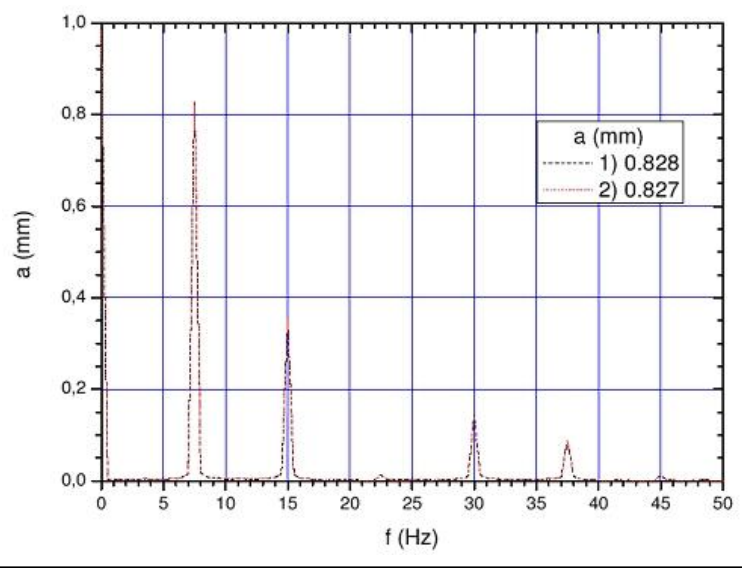


Even though one can estimate qualititavely the amplitude of vibration displacement from Fig. 4, it is not enough to describe the relationship between the amplitude and frequency. In this ocndition, Fast Fourier Transforms were applied to process the data, which gives the exact amplitude of the vibration as illustrated in Fig. 5. What also shown obviously in Fig. 5 is the vibrating frequency of the shaker, it is exactly equal to $7.5 \mathrm{~Hz}$, the driving frequency, and its higher order harmonics are clearly depicted.

During the study of Faraday instabilities, and later, the optical measurements of surface shape and the research of electric current and magnetic field distribution, it was found that there was some splashing of potassium hydroxide $(\mathrm{KOH})$ solution when the cylinder was vibrating vertically. Since $\mathrm{KOH}$ solution is corrosive to the contacting displacement meter which is made of aluminium alloy, the contacting meter was not applicable any longer, but a non-contacting device, a single-point Laser-Doppler Vibrometer (LDV) was exploited instead. In comparison with the contacting displacement meter, LDV cannot only give the information of displacements of the shaker, but also the vibration velocity.

Typical magnitudes of the base in 5 seconds were depicted in Fig. 6. As can be seen, two vertical straight lines were plotted to mark one period of the vibration which is given to be $0.1357 \mathrm{~s}$, i.e., equivalent to a vibrating frequency of $7.31 \mathrm{~Hz}$ lying in between $7.2 \mathrm{~Hz}$ and7.6 $\mathrm{Hz}$ at which a stable Faraday instability was observed. Besides, the peak values of the velocity are both $1.12 \mathrm{~m} / \mathrm{s}$, which indicate a perfect periodical oscillation.

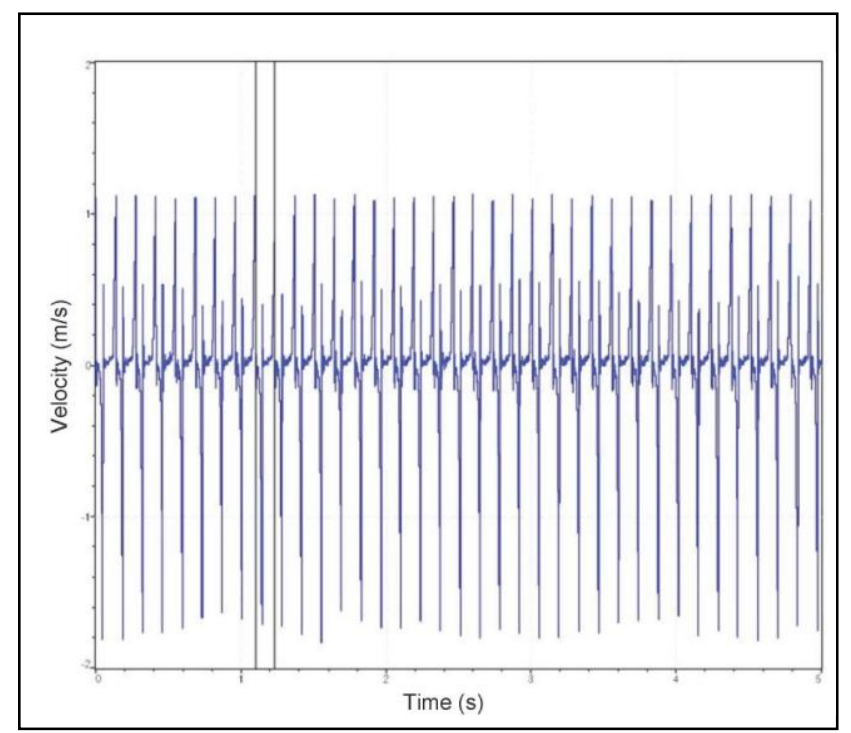

Figure 6 Velocity of the base measured by the Laser-Doppler vibrometer

Although the displacement cannot be measured directly by the Laser-Doppler vibrometer, it can be computed by integrating the measured data of velocity. It was carried out by a software Vibsoft provided by the manufacturer of the vibrometer. However, it is a little difficult to estimate the precise value of the displacement since there is a

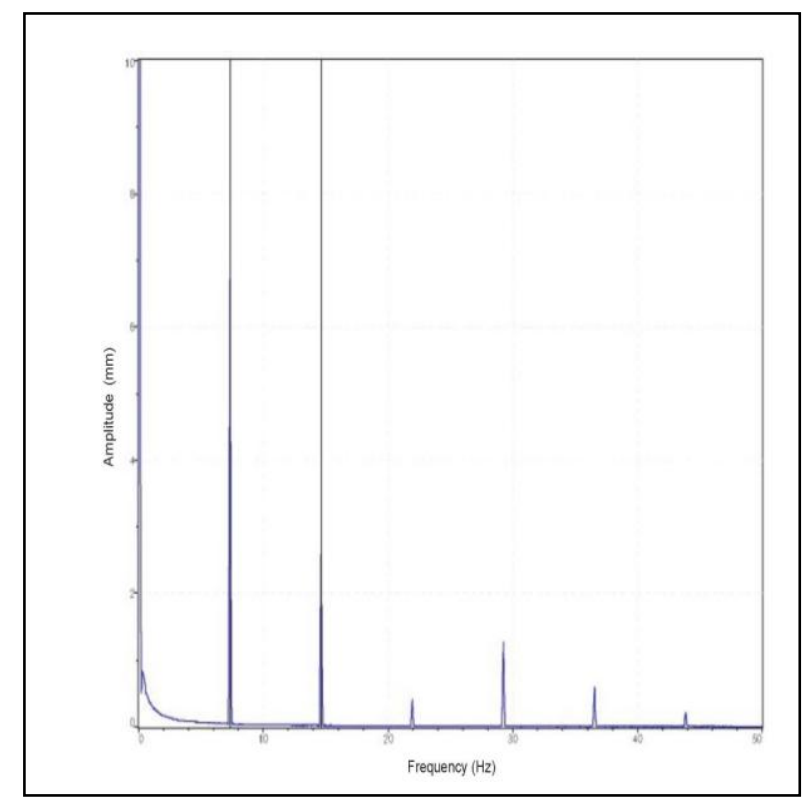

Figure 7 FFT of displacement integrated from velocity data

vertical shift with time, which is caused by a non-zero offset of the velocity. Fast Fourier transforms were applied again to process the data, the FFT result is given in Fig. 5, the magnitude of the first peak is $7.01 \mathrm{~mm}$ at a frequency of $7.31 \mathrm{~Hz}$, and the second peak locates at $14.63 \mathrm{~Hz}$, which is quantitatively consistent with the results obtained by the contacting displacement meter.

\section{CONCLUSIONS}

In the model experiment to investigate Faraday instabilities excited by a pneumatic shaker, a shock absorber and the combination of the shock absorber and a rubber belt was exploited to control the amplitude of the vibration displacement, respectively. It is found that there are some large fluctuations in the displacement if the shock absorber was used uniquely whereas the fluctuations were reduced dramatically when the shock absorber and a rubber belt were applied simultaneously. Besides, a contacting displacement meter can be exploited to measure the displacement of the solid shaker base with a resolution of $1 \mu \mathrm{m}$. However, if there are some corrosive solutions splashing outside of the cylinder, the contacting displacement meter is no longer applicable but a singlepoint Fibre-Optic Laser Vibrometer is appropriate to measure the velocity of the vibration, and the vibration displacement can be ontained by an integral to the velocity data.

\section{ACKNOWLEDGMENT}

This work was supported by Scientific Research Foundation of Education Department of Shaanxi Province (grant number 11JK0518). 


\section{REFERENCES}

[1] Thorleif Sele, "Computer model for magnetic field in electrolyttic cells including the effect of steel parts", Metallurgical Transactions, 5, 1974, pp. 2145-2150.

[2] Thorleif Sele, "Instabilities of the metal surface in electrolytic alumina reduction cells", Metallurgical Transactions B, 8B, 1977, pp. 613-618.

[3] Donald P. Ziegler, "Stability of metal/electrolyte interface in HallHeroult cells: effect of the steady velocity", Metallurgical Transactions B, 24B, 1993, pp. 899-906.

[4] Anderson P A, “An energy analysis of unstable, aluminium reduction cell”, Eur. J. Mech. B/fluids, 13, no 1, 1994, pp. 15-32.

[5] Anderson P A, "Magnetohydrodynamics in materials processing", Annual Review of Fluid Mechanics, vol. 31, 1999, pp. 273-300.

[6] Oleg Zikanov, Andre Thess, P.A. Davidson, and Donald P. Ziegler, "A New Approach to Numerical Simulation of Melt Flows and
Interface Instability in Hall-He'roult Cells", Metallurgical and Materials Transactions B, 31B, 2000, pp. 1541-1550.

[7] Baumgartl J, Hubert A, and Müller G, "The use of magnetohydrodynamic effects to investigate fluid flow in electrically conducting melts", Physics of Fluids, A 5, 1993, pp. 3280-3289.

[8] Stefani F, and Gerbeth G, "Velocity reconstruction in conducting fluids from magnetic field and electric potential measurements", Inverse Problems vol. 15, 1999, pp. 771-786.

[9] Festo cylinder with piston rod, http://www.festo.com/cms/nlbe_be/9725.htm

[10] Pedro Luís Andrighetto, : “Antonio Carlos Valdiero, Leonardo Carlotto. Study of the friction behavior in industrial pneumatic actuators " , ABCM Symposium Series in Mechatronics, 2, 2006, pp. 369-376. 